\title{
Specht's invariant and localization of operator tuples
}

\author{
Li Chen
}

\begin{abstract}
We introduce a set of operator-valued invariants for localization of operator tuples in the Cowen-Douglas class, with which a Specht-type criterion for unitary equivalence is obtained.

Key words: Specht's invariant; localization; Cowen-Douglas operator
\end{abstract}

\section{Introduction}

Finding suitable invariants to classify non-normal Hilbert space operators up to unitary equivalence is in general a widely open and appealing topic. A basic existence theorem of scaler-valued invariants for finite matrices was given by Specht in terms of matrix traces:

Theorem 1.1. (Specht [19]) Two complex $n \times n$ matrices $A$ and $B$ are unitarily equivalent if and only if $\operatorname{tr}\left(w\left(A, A^{*}\right)\right)=\operatorname{tr}\left(w\left(B, B^{*}\right)\right)$ for all words $w$ in two variables.

On infinite dimensional Hilbert spaces where scaler invariants are not always obtainable, a natural idea is to consider operator-valued alternatives. By operator-valued invariants we mean that one associates to every operator $T$ (in a given operator class) a set $\mathcal{I}(T)$ consisting of "testing operators" acting on certain "testing spaces", such that unitary equivalence of $T$ can be reduced to unitary equivalences of operators in $\mathcal{I}(T)$. A well-known example [16] is the complete non-unitary contractions, whose unitary equivalence can be determined by the so-called characteristic function as a family of testing operators parameterized by points in the unit disc acting on the defect spaces of the contractions.

Remark 1.2. Operator-valued invariants always exist(one can trivially set $\mathcal{I}(T)=\{T\}$ as a singleton with entire space as the testing space), and conceptual non-triviality lies in the existence of small and canonical testing spaces (in particular, existence of scaler invariants follows from existence of one dimensional testing spaces). Minimizing the cardinality of $\mathcal{I}(T)$, after testing spaces identified and fixed, is a technical problem (see, for instance, a series of refinements [17, 18, 10] on Theorem [1.1) and will not be the theme of this paper.

In this paper we work on multi-variate case with a tuple of commuting operators lying in the following important and extensively studied class introduced by M. Cowen and R.Douglas [3, 4]. 
Definition 1.3. Given positive integers $m, n$, and a bounded domain $\Omega$ in $\mathbb{C}^{m}$, a commuting $m$-tuple of operators $\boldsymbol{T}=\left(T_{1}, \cdots, T_{m}\right)$ acting on a Hilbert space $\mathcal{H}$ belongs to the class $\mathcal{B}_{n}^{m}(\Omega)$ if the followings hold:

(i) The space $\left\{\left(\left(T_{1}-z_{1}\right) h, \cdots,\left(T_{m}-z_{m}\right) h\right), h \in \mathcal{H}\right\}$ is a closed subspace in $\mathcal{H} \oplus \cdots \oplus \mathcal{H}(m$ copies of $\mathcal{H})$ for every $\boldsymbol{z}=\left(z_{1}, \cdots, z_{m}\right) \in \Omega$;

(ii) $\operatorname{dim} \cap_{i=1}^{m} \operatorname{ker}\left(T_{i}-z_{i}\right)=n$ for every $\boldsymbol{z} \in \Omega$ and

(iii) $\vee_{z \in \Omega} \cap_{i=1}^{m} \operatorname{ker}\left(T_{i}-z_{i}\right)=\mathcal{H}$ (here $\vee$ denotes the closed linear span).

Operator tuples in $\mathcal{B}_{n}^{m}(\Omega)$ can be modeled by adjoints of coordinate multiplications on $\mathbb{C}^{n}$-valued holomorphic function spaces in $m$ complex variables [5] such as Hardy or Bergman spaces, making it a rich class of considerable interest. We record the following theorem which is the main result of Cowen and Douglas' seminal work, asserting that unitary equivalence of operator tuples in $\mathcal{B}_{n}^{m}(\Omega)$ can be tested on a family of finite dimensional spaces.

Theorem 1.4. ([2, 3]) Operator tuples $\mathbf{T}$ and $\widetilde{\mathbf{T}}$ in $\mathcal{B}_{n}^{m}(\Omega)$ are unitarily equivalent if and only if $\left.\mathbf{T}\right|_{H_{z}^{n+1}}$ is unitarily equivalent to $\left.\widetilde{\mathbf{T}}\right|_{\widetilde{H}_{z}^{n+1}}$ for every $\boldsymbol{z}$ in $\Omega$.

Here the space

$$
H_{\mathbf{z}}^{k}:=\bigcap_{|I|=k} \operatorname{ker}(\mathbf{T}-\mathbf{z})^{I}
$$

is called the $k$-th order localization of $\mathbf{T}$ at $\mathbf{z}=\left(z_{1}, \cdots, z_{m}\right)$, where

$$
(\mathbf{T}-\mathbf{z})^{I}:=\left(T_{1}-z_{1}\right)^{i_{1}}\left(T_{2}-z_{2}\right)^{i_{2}} \cdots\left(T_{m}-z_{m}\right)^{i_{m}}
$$

$I=\left(i_{1}, \cdots, i_{m}\right)$ and $|I|=i_{1}+\cdots+i_{m}$.

Theorem 1.4 as a surprising result is well-known for its purely geometric proof(see Sec 3 , [3]) via a holomorphic vector bundle $E(\mathbf{T})$ over $\Omega$ associated to $\mathbf{T}$, where the fiber of $E(\mathbf{T})$ at $\mathbf{z}$ is nothing but the first order localization $H_{\mathbf{z}}^{1}=\cap_{i=1}^{m} \operatorname{ker}\left(T_{i}-z_{i}\right)$ (see Proposition 2.1 below). Geometrically reducing unitary equivalence of $\left.\mathbf{T}\right|_{H_{\mathbf{z}}^{n+1}}$ to criterions involving the curvature of $E(\mathbf{T})$ and its covariant derivatives living on the fiber $H_{\mathbf{z}}^{1}$ is not only a key step in the proof of Theorem 1.4(see Sec 2, [3]), but also motivates numerous interesting later works, including the study of flag structure and curvature inequalities of $E(\mathbf{T})$ that are of independent geometric interest $([4,11,12,13,14,15$, 20] $)$, as well as geometric classification theories for Hilbert modules([1, 6, 7, 8, 9]).

In this paper we show that unitary equivalence of $H_{\mathbf{z}}^{k}$ can be determined by a set of Specht-type operators (involving words in $T_{1}, \cdots, T_{m}$ and $T_{1}^{*}, \cdots, T_{m}^{*}$ ) acting on $H_{\mathbf{z}}^{1}$, which is an operator-theoretic counterpart of the above mentioned geometric reduction and extends results from recent works of Misra, Pal and Reza([14, 15]) as well. In particular, as the first order localization is much smaller than high order ones, letting $\mathbf{z}$ run through $\Omega$ our result (applied to localizations of order $n+1$ ) implies a refinement of Theorem 1.4 in light of Remark 1.2. 


\section{Prelimineries}

For a separable Hilbert space $\mathcal{H}$ and a positive integer $n$, let $\mathcal{G} r(n, \mathcal{H})$ denote the Grassmann manifold of all $n$-dimensional subspaces of $\mathcal{H}$. A map $E: \Omega \rightarrow \mathcal{G} r(n, \mathcal{H})$ is called a holomorphic curve if there exists $n$ holomorphic $\mathcal{H}$-valued functions $\gamma_{1}, \cdots, \gamma_{n}$, called a holomorphic frame, such that $E(\mathbf{z})=\operatorname{span}\left\{\gamma_{1}(\mathbf{z}), \cdots, \gamma_{n}(\mathbf{z})\right\}$ for every $\mathbf{z} \in \Omega$. In particular, this defines a holomorphic Hermitian vector bundle of rank $n$ over $\Omega$ whose Hermitian metric is just the inner product of $\mathcal{H}$. The following important observation of Cowen and Douglas(Proposition 1.11, [3]) asserts that the family of joint eigen-spaces of an operator tuple $\mathbf{T} \in \mathcal{B}_{n}^{m}(\Omega)$ is such an bundle.

Proposition 2.1. For any $\mathbf{T} \in \mathcal{B}_{n}^{m}(\Omega)$, the map $\boldsymbol{z} \mapsto \cap_{i=1}^{m} \operatorname{ker}\left(T_{i}-z_{i}\right)$ defines a holomorphic curve.

We denote this holomorphic curve by $E(\mathbf{T})$. In particular, there exists holomorphic $\mathcal{H}$-valued functions $\gamma_{1}, \cdots, \gamma_{n}$ over $\Omega$ such that $\cap_{i=1}^{m} \operatorname{ker}\left(T_{i}-z_{i}\right)=\operatorname{span}\left\{\gamma_{1}(\mathbf{z}), \cdots, \gamma_{n}(\mathbf{z})\right\}$. Now we further represent the high order localization $H_{\mathbf{z}}^{k}=\bigcap_{|I|=k} \operatorname{ker}(\mathbf{T}-\mathbf{z})^{I}$ in terms of derivatives of these functions.

For a fixed multi-index $I=\left(i_{1}, \cdots, i_{m}\right)$, set $I !=i_{1} ! \cdots i_{m}$ ! and $\partial^{I}=\partial_{1}^{i_{1}} \partial_{2}^{i_{2}} \cdots \partial_{m}^{i_{m}}$ where $\partial_{i}$ denotes differentiation with respect to $z_{i}, 1 \leq i \leq m$. Given another multi-index $J=\left(j_{1}, \cdots, j_{m}\right)$, we say $I \geq J$ if $i_{k} \geq j_{k}$ for all $1 \leq k \leq m$, and the index $\left(i_{1}-j_{1}, \cdots, i_{m}-j_{m}\right)$ is denoted by $I-J$.

As $\cap_{i=1}^{m} \operatorname{ker}\left(T_{i}-z_{i}\right)=\operatorname{span}\left\{\gamma_{1}(\mathbf{z}), \cdots, \gamma_{n}(\mathbf{z})\right\}$, the identity

$$
\left(T_{j}-z_{j}\right) \gamma_{i}(\mathbf{z})=0
$$

holds for every $1 \leq i, j \leq m$, which, combined with standard differentiation computations via the Leibnitz rule(or see p.470, [5]), yields

$$
(\mathbf{T}-\mathbf{z})^{I} \partial^{J} \gamma_{i}(\mathbf{z})= \begin{cases}\frac{J !}{(J-I) !} \gamma_{i}(\mathbf{z}), J \geq I \\ 0, & \text { otherwise }\end{cases}
$$

Lemma 2.2. Given an operator tuple $\mathbf{T}=\left(T_{1}, \cdots, T_{m}\right) \in \mathcal{B}_{n}^{m}(\Omega)$ and a fixed holomorphic frame $\left\{\gamma_{1}, \cdots, \gamma_{n}\right\}$ for $E(\mathbf{T})$, it holds that for any positive integer $k$,

$$
\cap_{|I|=k} \operatorname{ker}(\mathbf{T}-\boldsymbol{z})^{I}=\operatorname{span}_{|I| \leq k-1}\left\{\partial^{I} \gamma_{i}(\mathbf{z}), 1 \leq i \leq n\right\}
$$

Proof. That $\operatorname{span}_{|I| \leq k-1}\left\{\partial^{I} \gamma_{i}(\mathbf{z}), 1 \leq i \leq n\right\} \subseteq \cap_{|I|=k} \operatorname{ker}(\mathbf{T}-\mathbf{z})^{I}$ trivially follows from (2.1) and we prove $\cap_{|I|=k} \operatorname{ker}(\mathbf{T}-\mathbf{z})^{I} \subseteq \operatorname{span}_{|I| \leq k-1}\left\{\partial^{I} \gamma_{i}(\mathbf{z}), 1 \leq i \leq n\right\}$ by induction.

The conclusion trivially holds when $k=1$ and we suppose it holds for some $k$. Now fix $x \in \cap_{|I|=k+1} \operatorname{ker}(\mathbf{T}-\mathbf{z})^{I}$, then for any $I$ such that $|I|=k,(\mathbf{T}-\mathbf{z})^{I} x$ lies in $\cap_{|I|=1} \operatorname{ker}(\mathbf{T}-\mathbf{z})^{I}$, 
which is the joint eigen-space spanned by $\left\{\gamma_{1}(\mathbf{z}), \cdots, \gamma_{n}(\mathbf{z})\right\}$. Hence we get a collection of complex numbers $\left\{a_{i}^{J}|1 \leq i \leq n| J \mid,=k\right\}$ such that

$$
(\mathbf{T}-\mathbf{z})^{I} x=\sum_{i=1}^{n} a_{i}^{I} \gamma_{i}(\mathbf{z})
$$

whenever $|I|=k$.

We claim that the vector

$$
x-\sum_{|J|=k} \sum_{i=1}^{n} \frac{a_{i}^{J}}{J !} \partial^{J} \gamma_{i}(\mathbf{z})
$$

lies in $\cap_{|I|=k} \operatorname{ker}(\mathbf{T}-\mathbf{z})^{I}$. Then the induction hypothesis together with the claim implies that $x \in \operatorname{span}_{|J| \leq k}\left\{\partial^{J} \gamma_{i}(\mathbf{z}), 1 \leq i \leq n\right\}$, which gives the conclusion for $k+1$ and completes the induction.

To verify the claim, we fix a multi-index $I$ such that $|I|=k$, then for any multi-index $J$ with $|J|=k$, it holds by (2.1) that

$$
(\mathbf{T}-\mathbf{z})^{I} \partial^{J} \gamma_{i}(\mathbf{z})=\left\{\begin{array}{l}
I ! \gamma_{i}(\mathbf{z}), I=J \\
0, \quad I \neq J
\end{array}\right.
$$

This implies

$$
(\mathbf{T}-\mathbf{z})^{I} \sum_{|J|=k} \sum_{i=1}^{n} \frac{a_{i}^{J}}{J !} \partial^{J} \gamma_{i}(\mathbf{z})=(\mathbf{T}-\mathbf{z})^{I} \sum_{i=1}^{n} \frac{a_{i}^{I}}{I !} \partial^{I} \gamma_{i}(\mathbf{z})=\sum_{i=1}^{n} a_{i}^{I} \gamma_{i}(\mathbf{z})
$$

and the claim follows by comparing (2.2) and (2.3).

We end this section with two elementary lemmas.

Lemma 2.3. Let $E$ be a holomorphic curve over $\Omega$ of rank $n$. For any point $\boldsymbol{z}_{0}$ in $\Omega$, there exist a holomorphic frame $\left\{\gamma_{i}(\boldsymbol{z})\right\}_{i=1}^{n}$ for $E$ over an neighborhood of $\boldsymbol{z}_{0}$ on which $\left\langle\gamma_{i}(\boldsymbol{z}), \gamma_{j}\left(\boldsymbol{z}_{0}\right)\right\rangle=\delta_{i j}$ for all $1 \leq i, j \leq n$, where $\delta_{i j}$ is the Kronecker symbol.

Lemma 2.4. Let $f(\boldsymbol{z}, \boldsymbol{w})$ be a function on $\Omega \times \Omega$ which is holomorphic in $\boldsymbol{z}$ and antiholomorphic in $\boldsymbol{w}$. If $f(\boldsymbol{z}, \boldsymbol{z})=0$ for all $\boldsymbol{z} \in \Omega$, then $f(\boldsymbol{z}, \boldsymbol{w})$ vanishes identically on $\Omega \times \Omega$.

Lemma 2.3(see Lemma 2.4, 3]) asserts that a holomorphic curve always admits a holomorphic frame normalized at a single point which is called a normalized frame, and Lemma 2.4 is standard which will be useful in dealing with normalized frames later. 


\section{Main result}

Throughout this section, $k$ will be a fixed positive integer. We show that for an operator tuple $\mathbf{T}=\left(T_{1}, \cdots, T_{m}\right) \in \mathcal{B}_{n}^{m}(\Omega)$, unitary equivalence of its $k$-th order localization $\mathbf{T}_{H_{\mathbf{z}}^{k}}$ can be tested by a collection of "Specht-type" operators acting on the first order localization $H_{\mathrm{z}}^{1}=\cap_{i=1}^{m} \operatorname{ker}\left(T_{i}-z_{i}\right)$.

Precisely, for each multi-index $I=\left(i_{1}, \cdots, i_{m}\right)$, and $\mathbf{z}=\left(z_{1}, \cdots, z_{m}\right) \in \Omega$, set

$$
N_{\mathbf{z}}^{I}:=\left.\left(T_{1}-z_{1}\right)^{i_{1}}\left(T_{2}-z_{2}\right)^{i_{2}} \cdots\left(T_{m}-z_{m}\right)^{i_{m}}\right|_{H_{\mathbf{z}}^{k}}
$$

and for two fixed multi-indices $I, J$, set

$$
K_{\mathbf{z}}^{I J}:=\left.P_{H_{\mathbf{z}}^{1}}\left[N_{\mathbf{z}}^{I}\left(N_{\mathbf{z}}^{J}\right)^{*}\right]\right|_{H_{\mathbf{z}}^{1}}
$$

Here as $N_{\mathbf{z}}^{I}\left(N_{\mathbf{z}}^{J}\right)^{*}$ does not necessarily leave $H_{\mathbf{z}}^{1}$ invariant, $P_{H_{\mathbf{z}}^{1}}$ is imposed to make $K_{\mathbf{z}}^{I J}$ live in $H_{\mathbf{z}}^{1}$.

The main result of this paper as follows gives the Specht-type classification for $\mathbf{T}_{H_{\mathbf{z}}^{k}}$ in terms of $K_{\mathbf{z}}^{I J}$ :

Theorem 3.1. Given operator tuples $\mathbf{T}$ and $\widetilde{\mathbf{T}}$ in $\mathcal{B}_{n}^{m}(\Omega)$, their localizations $\left.\mathbf{T}\right|_{H_{\mathbf{z}}^{k}}$ and $\left.\widetilde{\mathbf{T}}\right|_{\widetilde{H}_{\mathbf{z}}^{k}}$ are unitarily equivalent if and only if operator tuples $\left\{K_{z}^{I J}, 1 \leq|I|,|J| \leq k-1\right\}$ and $\left\{\widetilde{K}_{z}^{I J}, 1 \leq|I|,|J| \leq k-1\right\}$ are unitarily equivalent.

The first nontrivial case $k=2$ (where one can check that $H_{\mathbf{z}}^{1}$ is actually invariant under $\left.N_{\mathbf{z}}^{I}\left(N_{\mathbf{z}}^{J}\right)^{*}\right)$ were studied by Misra, Pal and Reza in their works on curvature inequalities for $\mathcal{B}_{n}^{m}(\Omega)\left[14\right.$, 15]. If we assume further that $n=1$, then $K_{\mathbf{z}}^{I J}$ as a scaler exactly equals the trace of $N_{\mathbf{z}}^{I}\left(N_{\mathbf{z}}^{J}\right)^{*}$ so it is eligible to call $K_{\mathbf{z}}^{I J}$ a Specht-type invariant.

With Theorem 3.1, we immediately get the following refinement of Theorem 1.4

Theorem 3.2. Operator tuples $\mathbf{T}$ and $\widetilde{\mathbf{T}}$ in $\mathcal{B}_{n}^{m}(\Omega)$ are unitarily equivalent if and only if operator tuples $\left\{K_{z}^{I J}, 1 \leq|I|,|J| \leq n\right\}$ and $\left\{\widetilde{K}_{z}^{I J}, 1 \leq|I|,|J| \leq n\right\}$ are unitarily equivalent for every $\boldsymbol{z}$ in $\Omega$.

The proof of Theorem 3.1 will be given in the end of this section after some preparations. Before proceeding, we fix some notations and conventions in elementary linear algebra.

(i) "Inner product" of matrices: Let $A=\left[a_{i j}\right]_{m \times n}$ and $B=\left[b_{i j}\right]_{n \times p}$ be two matrices with entries $a_{i j}, b_{i j}$ lying in a Hilbert space(whose inner product is denoted by $\langle$,$\rangle ). Let$ $\langle A, B\rangle$ denotes the numerical matrix $E=\left[e_{i j}\right]_{m \times p}$ given by $e_{i j}=\sum_{k=1}^{n}\left\langle a_{i k}, b_{k j}\right\rangle$. If $C, D$ are numerical matrices, then $\langle C A, B D\rangle=C\langle A, B\rangle \bar{D}$.

With this notation, if $\gamma=\left(\gamma_{1}, \cdots, \gamma_{n}\right)$ is a holomorphic frame for a rank $n$ holomorphic curve, then its Gram matrix can be written as $\left\langle\gamma^{T}(\mathbf{z}), \gamma(\mathbf{z})\right\rangle$. Moreover, $\gamma$ is normalized at a point $\mathbf{z}_{0}$ if and only if $\left\langle\gamma^{T}(\mathbf{z}), \gamma\left(\mathbf{z}_{0}\right)\right\rangle=I$ identically. 
(ii) Representation of linear maps: We adopt the "left action" convention regarding to representing matrices for linear maps. Precisely, let $\Phi$ be a linear map on a linear space spanned by $\gamma=\left(\gamma_{1}, \cdots, \gamma_{n}\right)$, then a matrix $A=\left[a_{i j}\right]$ represents $\Phi$ if $\Phi \gamma_{i}=\sum a_{i j} \gamma_{j}$, or in other words, $\Phi \gamma^{T}=\left(\Phi \gamma_{1}, \cdots, \Phi \gamma_{n}\right)^{T}=A\left(\gamma_{1}, \cdots, \gamma_{n}\right)^{T}$. If another linear map $\Psi$ is represented by $B=\left[b_{i j}\right]$, then $\Phi \Psi$ is represented by $B A$ (not $A B$, which corresponds to "right action" convention).

Throughout this section we will work with normalized frames of $E(\mathbf{T})$. In the single variable case $m=1$, the normalized frame was used in the study of geometric theory of $\mathcal{B}_{n}^{1}(\Omega)$ to identify localization order of an operator with "contact order" of a holomorphic curve(see Section 2, [3] for details), and here we need the following variation for $\mathcal{B}_{n}^{m}(\Omega)$ as a preparation before proving Theorem 3.1 .

Theorem 3.3. The followings are equivalent

(i) $H_{\mathbf{z}}^{k}$ and $\widetilde{H}_{\mathbf{z}}^{k}$ are unitarily equivalent;

(ii)there exists holomorphic frames $\gamma$ and $\widetilde{\gamma}$ (whose Gram matrices are denoted by $H$ and $\widetilde{H})$ for $E(\boldsymbol{T})$ and $E(\widetilde{\boldsymbol{T}})$ such that $\partial^{I} \bar{\partial}^{J} H=\partial^{I} \bar{\partial}^{J} \widetilde{H}$ at $\mathbf{z}$ for all $|I|,|J| \leq k-1$;

(iii)there exists holomorphic frames $\gamma$ and $\widetilde{\gamma}$ normalized at $\mathbf{z}$ such that $\partial^{I} \bar{\partial}^{J} H=\partial^{I} \bar{\partial}^{J} \widetilde{H}$ at $\mathbf{z}$ for all $|I|,|J| \leq k-1$;

(iv) there exists holomorphic frames $\gamma$ and $\widetilde{\gamma}$ normalized at $\mathbf{z}$ and a constant unitary matrix $U$, such that $\partial^{I} \bar{\partial}^{J} H=U\left(\partial^{I} \bar{\partial}^{J} \widetilde{H}\right) U^{*}$ at $\mathbf{z}$ for all $|I|,|J| \leq k-1$;

(v) For any holomorphic frames $\gamma$ and $\widetilde{\gamma}$ normalized at $\mathbf{z}$, there exists a constant unitary matrix $U$, such that $\partial^{I} \bar{\partial}^{J} H=U\left(\partial^{I} \bar{\partial}^{J} \widetilde{H}\right) U^{*}$ at $\mathbf{z}$ for all $|I|,|J| \leq k-1$.

We need two elementary lemmas before the proof of Theorem 3.3 and Theorem 3.1 .

Lemma 3.4. Let $\boldsymbol{\gamma}=\left\{\gamma_{1}, \cdots, \gamma_{n}\right\}$ and $\boldsymbol{\beta}=\left\{\beta_{1}, \cdots, \beta_{n}\right\}$ be two holomorphic frames of a holomorphic curve over $\Omega$ such $\boldsymbol{\gamma}$ is normalized at a point $\boldsymbol{z}_{0}$. Then $\boldsymbol{\beta}$ is normalized at $\boldsymbol{z}_{0}$ if and only if its transition function with $\gamma$ is a constant unitary matrix.

Proof. For one direction, let $U$ be a constant unitary matrix and $\boldsymbol{\beta}^{T}=U \boldsymbol{\gamma}^{T}$, then for $\mathbf{z}$ in $\Omega$,

$$
\left\langle\boldsymbol{\beta}^{T}(\mathbf{z}), \boldsymbol{\beta}(\mathbf{z})\right\rangle=U\left\langle\boldsymbol{\gamma}^{T}(\mathbf{z}), \boldsymbol{\gamma}(\mathbf{z})\right\rangle U^{*}
$$

where $U^{*}$ denotes the conjugate transpose of $U$. The above identity can be refined by Lemma 2.4 into

$$
\left\langle\boldsymbol{\beta}^{T}(\mathbf{z}), \boldsymbol{\beta}(\mathbf{w})\right\rangle=U\left\langle\boldsymbol{\gamma}^{T}(\mathbf{z}), \boldsymbol{\gamma}(\mathbf{w})\right\rangle U^{*}
$$

for all $\mathbf{z}, \mathbf{w} \in \Omega$. As $\gamma$ is normalized at $\mathbf{z}_{0},\left\langle\gamma^{T}(\mathbf{z}), \gamma\left(\mathbf{z}_{0}\right)\right\rangle=I$, hence by setting $\mathbf{w}=\mathbf{z}_{0}$ the above equation becomes

$$
\left\langle\boldsymbol{\beta}^{T}(\mathbf{z}), \boldsymbol{\beta}\left(\mathbf{z}_{0}\right)\right\rangle=U\left\langle\boldsymbol{\gamma}^{T}(\mathbf{z}), \gamma\left(\mathbf{z}_{0}\right)\right\rangle U^{*}=U U^{*}=I
$$


Hence $\boldsymbol{\beta}$ is also normalized at $\mathbf{z}_{0}$.

Conversely, let $U(\mathbf{z})$ be the transition function of $\boldsymbol{\beta}$ with $\boldsymbol{\gamma}$, then $U(\mathbf{z})$ is holomorphic and

$$
\left\langle\boldsymbol{\beta}^{T}(\mathbf{z}), \boldsymbol{\beta}(\mathbf{z})\right\rangle=U(\mathbf{z})\left\langle\gamma^{T}(\mathbf{z}), \boldsymbol{\gamma}(\mathbf{z})\right\rangle U(\mathbf{z})^{*},
$$

which can be refined into

$$
\left\langle\boldsymbol{\beta}^{T}(\mathbf{z}), \boldsymbol{\beta}(\mathbf{w})\right\rangle=U(\mathbf{z})\left\langle\gamma^{T}(\mathbf{z}), \boldsymbol{\gamma}(\mathbf{w})\right\rangle U(\mathbf{w})^{*} .
$$

If $\boldsymbol{\beta}$ is also normalized at $\mathbf{z}_{0}$, then $\left\langle\boldsymbol{\beta}^{T}(\mathbf{z}), \boldsymbol{\beta}\left(\mathbf{z}_{0}\right)\right\rangle=\left\langle\boldsymbol{\gamma}^{T}(\mathbf{z}), \boldsymbol{\gamma}\left(\mathbf{z}_{0}\right)\right\rangle=I$ and the above equation becomes(by setting $\mathbf{w}=\mathbf{z}_{0}$ )

$$
I=\left\langle\boldsymbol{\beta}^{T}(\mathbf{z}), \boldsymbol{\beta}\left(\mathbf{z}_{0}\right)\right\rangle=U(\mathbf{z})\left\langle\boldsymbol{\gamma}^{T}(\mathbf{z}), \boldsymbol{\gamma}\left(\mathbf{z}_{0}\right)\right\rangle U\left(\mathbf{z}_{0}\right)^{*}=U(\mathbf{z}) U\left(\mathbf{z}_{0}\right)^{*} .
$$

This gives $U(\mathbf{z})=U^{-1}\left(\mathbf{z}_{0}\right)^{*}$, so $U(\mathbf{z})$ is a constant unitary matrix.

The following lemma on elementary linear algebra is standard and we omit the proof.

Lemma 3.5. Let $\Phi$ be a linear operator on a finite dimensional Hilbert space and $\boldsymbol{\gamma}=$ $\left\{\gamma_{1}, \cdots, \gamma_{n}\right\}$ be a base whose Gram matrix is $H$. If $\Phi$ is represented by a matrix $A$ with respect to $\boldsymbol{\gamma}$, then its adjoint operator is represented by $H A^{*} H^{-1}$.

Proof of Theorem 3.3 ;

Proof. (ii) $\Rightarrow$ (i) Write $\gamma=\left\{\gamma_{1}, \cdots, \gamma_{n}\right\}$ and $\widetilde{\gamma}=\left\{\widetilde{\gamma}_{1}, \cdots, \widetilde{\gamma}_{n}\right\}$, hence by Lemma (2.2), $H_{z}^{k}=\operatorname{span}_{|J| \leq k-1}\left\{\partial^{J} \gamma_{i}(\mathbf{z}), 1 \leq i \leq n\right\}$ and $\widetilde{H}_{z}^{k}=\operatorname{span}_{|J| \leq k-1}\left\{\partial^{J} \widetilde{\gamma}_{i}(\mathbf{z}), 1 \leq i \leq n\right\}$. Let $\Phi$ be the linear map from $H_{\mathbf{z}}^{k}$ to $\widetilde{H}_{\mathbf{z}}^{k}$ defined by

$$
\Phi \partial^{J} \gamma_{i}(\mathbf{z}):=\partial^{J} \widetilde{\gamma}_{i}(\mathbf{z}),|J| \leq k-1,
$$

then $\Phi$ implements a unitary equivalence between $H_{\mathbf{z}}^{k}$ and $\widetilde{H}_{\mathbf{z}}^{k}$.

In fact, $\Phi$ trivially intertwines $T_{l}-z_{l}$ and $\widetilde{T}_{l}-z_{l}\left(\right.$ hence intertwines $T_{l}$ and $\left.\widetilde{T}_{l}\right), 1 \leq l \leq m$ as their actions on $\partial^{I} \boldsymbol{\gamma}(\mathbf{z})$ and $\partial^{I} \widetilde{\boldsymbol{\gamma}}(\mathbf{z})$ follows the same rule (2.1). Moreover, $\partial^{I} \bar{\partial}^{J} H=$ $\left[\left\langle\partial^{I} \gamma_{i}, \partial^{J} \gamma_{j}\right\rangle\right]_{1 \leq i, j \leq n}$ and $\partial^{I} \bar{\partial}^{J} \widetilde{H}=\left[\left\langle\partial^{I} \widetilde{\gamma}_{i}, \partial^{J} \widetilde{\gamma}_{j}\right\rangle\right]_{1 \leq i, j \leq n}$ since the frames are holomorphic, hence the condition (ii) implies

$$
\left\langle\partial^{I} \gamma_{i}, \partial^{J} \gamma_{j}\right\rangle=\left\langle\partial^{I} \widetilde{\gamma}_{i}, \partial^{J} \widetilde{\gamma}_{j}\right\rangle
$$

for every $1 \leq i, j \leq n$ and $|I|,|J| \leq k-1$ at $\mathbf{z}$, so $\Phi$ is isometric as well.

(iii) $\Rightarrow$ (ii) Trivial.

(i) $\Rightarrow$ (iii) Let $\Phi$ be a unitary operator from $H_{\mathbf{z}}^{k}$ to $\widetilde{H}_{\mathbf{z}}^{k}$ which implements the unitary equivalence. We show that there exists holomorphic frames $\gamma$ and $\widetilde{\gamma}$ normalized at $\mathbf{z}$ such that 


$$
\Phi \partial^{I} \gamma_{i}(\mathbf{z})=\partial^{I} \widetilde{\gamma}_{i}(\mathbf{z})
$$

for all $|I| \leq k-1,1 \leq i \leq n$, and (iii) will follow since $\Phi$ is isometric.

We begin with arbitrary fixed holomorphic frames $\gamma=\left\{\gamma_{1}, \cdots, \gamma_{n}\right\}$ and $\widetilde{\gamma}=\left\{\widetilde{\gamma}_{1}, \cdots, \widetilde{\gamma}_{n}\right\}$ for $E(\mathbf{T})$ and $E(\widetilde{\mathbf{T}})$ normalized at $\mathbf{z}$. As $\Phi$ intertwines $T_{l}-z_{l}$ and $\widetilde{T}_{l}-z_{l}$, it maps the joint eigen-space of $\mathbf{T}$ spanned by $\gamma(\mathbf{z})$ to corresponding one of $\widetilde{\mathbf{T}}$ spanned by $\widetilde{\gamma}(\mathbf{z})$, hence there exists an $n \times n$ matrix $U$ such that

$$
\Phi \gamma^{T}(\mathbf{z})=U \widetilde{\gamma}^{T}(\mathbf{z})
$$

and $U$ is unitary since both $\gamma$ and $\widetilde{\gamma}$ are normalized at z. By Lemma [3.4, $U \widetilde{\gamma}^{T}$ is again a normalized frame at $\mathbf{z}$, so we can replace $\widetilde{\gamma}^{T}$ by $U \widetilde{\gamma}^{T}$ which gives (3.1) in case $|I|=0$.

Now we check (3.1) by induction on $|I|$. Suppose (3.1) holds with all $|I| \leq l$ for some $l$. For any $I$ with $|I|=l+1$, it holds that

$$
\left(\widetilde{T}_{q}-z_{q}\right)\left(\Phi \partial^{I} \gamma_{i}(\mathbf{z})-\partial^{I} \widetilde{\gamma}_{i}(\mathbf{z})\right)=0
$$

for every $1 \leq q \leq m$.

In fact, by the intertwining property of $\Phi$,

$$
\left(\widetilde{T}_{q}-z_{q}\right)\left(\Phi \partial^{I} \gamma_{i}(\mathbf{z})-\partial^{I} \widetilde{\gamma}_{i}(\mathbf{z})\right)=\Phi\left(T_{q}-z_{q}\right) \partial^{I} \gamma_{i}(\mathbf{z})-\left(\widetilde{T}_{q}-z_{q}\right) \partial^{I} \widetilde{\gamma}_{i}(\mathbf{z})
$$

Write $I=\left(i_{1}, \cdots, i_{q}, \cdots, i_{m}\right\}$, then in case $i_{q}=0$, both $\left(T_{q}-z_{q}\right) \partial^{I} \gamma_{i}(\mathbf{z})$ and $\left(\widetilde{T}_{q}-z_{q}\right) \partial^{I} \widetilde{\gamma}_{i}(\mathbf{z})$ vanishes hence (3.2) trivially holds. In case $i_{q} \geq 1,\left(T_{q}-z_{q}\right) \partial^{I} \gamma_{i}(\mathbf{z})=i_{q} \partial^{I^{\prime}} \gamma_{i}(\mathbf{z})$ and $\left(\widetilde{T}_{q}-z_{q}\right) \partial^{I} \gamma_{i}(\mathbf{z})=i_{q} \partial^{I^{\prime}} \widetilde{\gamma}_{i}(\mathbf{z})$ where $I^{\prime}=\left(i_{1}, \cdots, i_{q}-1, \cdots, i_{m}\right)$, hence (3.2) follows from the induction hypothesis.

Moreover, we observe that

$$
\left\langle\Phi \partial^{I} \gamma_{i}(\mathbf{z})-\partial^{I} \widetilde{\gamma}_{i}(\mathbf{z}), \widetilde{\gamma}_{j}(\mathbf{z})\right\rangle=0
$$

for every $1 \leq j \leq n$.

In fact, as $\Phi$ is isometric and the frames are normalized at $\mathbf{z}$, it holds that

$\left\langle\Phi \partial^{I} \gamma_{i}(\mathbf{z})-\partial^{I} \widetilde{\gamma}_{i}(\mathbf{z}), \widetilde{\gamma}_{j}(\mathbf{z})\right\rangle=\left\langle\Phi \partial^{I} \gamma_{i}(\mathbf{z}), \Phi \gamma_{j}(\mathbf{z})\right\rangle-\left\langle\partial^{I} \widetilde{\gamma}_{i}(\mathbf{z}), \widetilde{\gamma}_{j}(\mathbf{z})\right\rangle=\left\langle\partial^{I} \gamma_{i}(\mathbf{z}), \gamma_{j}(\mathbf{z})\right\rangle-\left\langle\partial^{I} \widetilde{\gamma}_{i}(\mathbf{z}), \widetilde{\gamma}_{j}(\mathbf{z})\right\rangle=0-0=0$

as desired.

Now (3.2) implies that $\Phi \partial^{I} \gamma_{i}(\mathbf{z})-\partial^{I} \widetilde{\gamma}_{i}(\mathbf{z})$ lies in $\cap_{i=1}^{m} \operatorname{ker}\left(\widetilde{T}_{i}-z_{i}\right)$ which is spanned by $\left\{\widetilde{\gamma}_{1}(\mathbf{z}), \cdots, \widetilde{\gamma}_{n}(\mathbf{z})\right\}$, hence (3.3) forces $\Phi \partial^{I} \gamma_{i}(\mathbf{z})-\partial^{I} \widetilde{\gamma}_{i}(\mathbf{z})=0$, concluding the induction.

(v) $\Rightarrow$ (iv) Trivial.

(iv) $\Rightarrow$ (iii) Let $\gamma, \widetilde{\gamma}$ and $U$ be as given by (iv), then $\widetilde{\boldsymbol{\sigma}}^{T}:=U \widetilde{\boldsymbol{\gamma}}^{T}$ is again a holomorphic frame for $E(\widetilde{\mathbf{T}})$ normalized at $\mathbf{z}$ by Lemma 3.4. Moreover,

$$
\partial^{I} \bar{\partial}^{J}\left\langle\boldsymbol{\sigma}^{T}, \boldsymbol{\sigma}\right\rangle=\partial^{I} \bar{\partial}^{J}\left(U\left\langle\widetilde{\gamma}^{T}, \widetilde{\gamma}\right\rangle U^{*}\right)=U\left(\partial^{I} \bar{\partial}^{J}\left\langle\widetilde{\boldsymbol{\gamma}}^{T}, \widetilde{\gamma}\right\rangle\right) U^{*}
$$


holds in a neighborhood of $\mathbf{z}$ which, specifying at $\mathbf{z}$, equals $\partial^{I} \bar{\partial}^{J}\left\langle\gamma^{T}, \gamma\right\rangle$ by (iv), so $\gamma$ and $\boldsymbol{\sigma}$ meets (iii).

(iii) $\Rightarrow(\mathrm{v})$ Fix holomorphic frames $\boldsymbol{\beta}$ and $\widetilde{\boldsymbol{\beta}}$ normalized at $\mathbf{z}$ with properties given by (iii), then for arbitrarily chosen holomorphic frames $\gamma$ and $\widetilde{\gamma}$ normalized at $\mathbf{z}$, their exists, by Lemma 3.4, constant unitary matrices $V$ and $\widetilde{V}$ such that $\gamma^{T}=V \boldsymbol{\beta}^{T}$ and $\widetilde{\boldsymbol{\gamma}}^{T}=\widetilde{V} \widetilde{\boldsymbol{\beta}}^{T}$, which gives

$$
\partial^{I} \bar{\partial}^{J}\left\langle\boldsymbol{\gamma}^{T}, \boldsymbol{\gamma}\right\rangle=V\left(\partial^{I} \bar{\partial}^{J}\left\langle\boldsymbol{\beta}^{T}, \boldsymbol{\beta}\right\rangle\right) V^{*}=V\left(\partial^{I} \bar{\partial}^{J}\left\langle\widetilde{\boldsymbol{\beta}}^{T}, \widetilde{\boldsymbol{\beta}}\right\rangle\right) V^{*}=V \widetilde{V}^{*}\left(\partial^{I} \bar{\partial}^{J}\left\langle\widetilde{\boldsymbol{\gamma}}^{T}, \widetilde{\boldsymbol{\gamma}}\right\rangle\right) \widetilde{V} V^{*}
$$

at $\mathbf{z}$. The proof is completed by taking $U=V \widetilde{V}^{*}$.

Finally we give the proof Theorem 3.1 .

Proof. We fix a holomorphic frame $\gamma=\left\{\gamma_{1}, \cdots, \gamma_{n}\right\}$ for $E(\mathbf{T})$ normalized at $\mathbf{z}$ and begin by calculating the matrix representation of $K_{\mathbf{z}}^{I J}$ with respect to the base $\gamma(\mathbf{z})$ of $H_{\mathbf{z}}^{1}$ (and $\widetilde{K}_{\mathbf{z}}^{I J}$ follows in the same way), which will be read out from the representing matrix for $N_{\mathbf{z}}^{I} N_{\mathbf{z}}^{J^{*}}$ with respect to the base $\left\{\partial^{K} \gamma_{i}(\mathbf{z}), 1 \leq i \leq n,|K| \leq k-1\right\}$ of $H_{\mathbf{z}}^{k}$.

Let $L$ be the cardinality of the multi-index set $\{K,|K| \leq k-1\}$, then $\operatorname{dim} H_{\mathrm{z}}^{k}=n L(L$ can be worked out via binomial coefficients but we do not need the precise value). The Gram matrix for $\left\{\partial^{K} \gamma_{i}(\mathbf{z}), 1 \leq i \leq n, \quad|K| \leq k-1\right\}$, denoted by $\mathbf{H}$, is an $L \times L$ block matrix $\left[H_{I J}\right]_{0 \leq|I|,|J| \leq k-1}$ in which each block is an $n \times n$ matrix $H_{I J}:=\left[\left\langle\partial^{I} \gamma_{i}(\mathbf{z}), \partial^{J} \gamma_{j}(\mathbf{z})\right\rangle\right]_{1 \leq i, j \leq n}$.

In principle, to precisely locate a particular block $H_{I J}$ in $\mathbf{H}$ one need to assign an ordering for the multi-indices, that is, a bijection $\sigma$ from the set $\{K,|K| \leq k-1\}$ to $\{0,1,2, \cdots, L-1\}$. From now on we fix a particular ordering(the lexicographic ordering for instance), then we can write

$$
\left[H_{I J}\right]_{0 \leq|I|,|J| \leq k-1}=\left[H_{\sigma I, \sigma J}\right]_{0 \leq \sigma I, \sigma J \leq L-1}=\left[H_{i j}\right]_{0 \leq i, j \leq L-1},
$$

where the terminology " $I$-th row/column" makes sense(which refers to " $\sigma(I)$-th row/column"), and we can freely use the above three representations in the sequel which will not cause confusion. In particular, we assume that $\sigma(0, \cdots, 0)=0$, so the block $H_{00}$ is the Gram matrix of $\gamma$.

By (2.1), for fixed index $J, N_{z}^{J}$ maps $\partial^{J} \gamma(\mathbf{z})$ to $J ! \gamma(\mathbf{z})$, while for $K \neq J$, linear representation of $N_{z}^{J} \partial^{K} \gamma(\mathbf{z})$ in terms of $\left\{\partial^{K} \gamma(\mathbf{z}), \quad|K| \leq k-1\right\}$ has no $\gamma(\mathbf{z})$-component. Therefore $N_{\mathbf{z}}^{J}$ has the following block matrix representation with respect to $\left\{\partial^{K} \boldsymbol{\gamma},|K| \leq k-1\right\}(\boldsymbol{\gamma}(\mathbf{z})$ appears in the 0 -th place when we arrange $\left\{\partial^{K} \gamma(\mathbf{z}),|K| \leq k-1\right\}$ into a column).

$$
N_{z}^{J}=\left(\begin{array}{cccc}
\mathbf{0} & & & \\
\vdots & & & \\
J ! I_{n} & \mathbf{0} & \cdots & \mathbf{0} \\
\vdots & & & \\
\mathbf{0} & & &
\end{array}\right) J-t h
$$


Here we have not written out all nonzero blocks in $N_{z}^{J}$, since the only thing we need later is that the $(J, 0)$ block $J ! I_{n}$ is the only nonzero block throughout the 0 -th column and $J$-th row.

As the frame $\boldsymbol{\gamma}$ is normalized at $\mathbf{z}$, it holds that

$$
H_{I 0}=\left[\left\langle\partial^{I} \gamma_{i}(\mathbf{z}), \gamma_{j}(\mathbf{z})\right\rangle\right]_{1 \leq i, j \leq n}=\mathbf{0}
$$

(similarly, $H_{0 I}=\mathbf{0}$ ) for all $1 \leq|I| \leq k-1$ and $H_{00}=I_{n}$. Therefore, the block matrix $\mathbf{H}$ is of the form

$$
\left(\begin{array}{cccc}
I_{n} & \mathbf{0} & \cdots & \mathbf{0} \\
\mathbf{0} & H_{11} & \cdots & H_{1, L-1} \\
\vdots & \vdots & & \vdots \\
\mathbf{0} & H_{L-1,1} & \cdots & H_{L-1, L-1}
\end{array}\right)
$$

which in turn implies that its inverse $\mathbf{G}=\left[G_{I J}\right]_{0 \leq|I|,|J| \leq k-1}=\left[G_{i j}\right]_{0 \leq i, j \leq L-1}$ is of the same form.

Now suppose $|I|,|J| \geq 1$, then by Lemma 3.5, $N_{\mathbf{z}}^{I} N_{\mathbf{z}}^{J^{*}}$ can be represented by

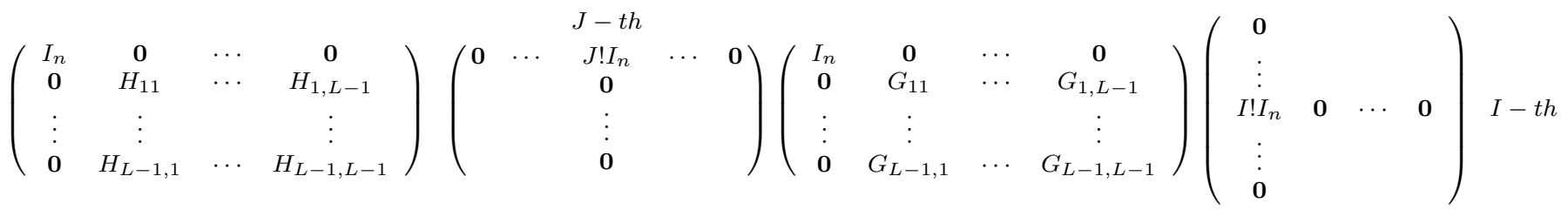

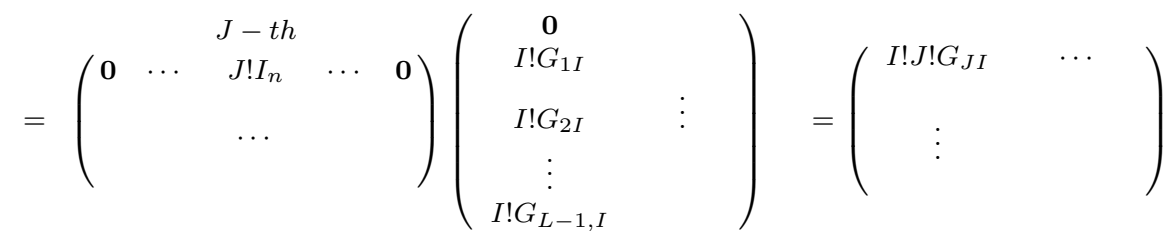

Since the frame is normalized at $\mathbf{z}$, the space $H_{\mathbf{z}}^{1}$ spanned by $\gamma(\mathbf{z})$ is is orthogonal to the space spanned by $\left\{\partial^{K} \gamma(\mathbf{z}), 1 \leq|K| \leq k-1\right\}$, which implies that the block $I ! J ! G_{J I}$ appearing at the left upper corner of $N_{\mathbf{z}}^{I} N_{\mathbf{z}}^{J^{*}}$ exactly represents $\left.P_{H_{\mathbf{z}}^{1}}\left(N_{\mathbf{z}}^{I} N_{\mathbf{z}}^{J^{*}}\right)\right|_{H_{\mathbf{z}}^{1}}$ with respect to the base $\gamma(\mathbf{z})$ of $H_{\mathbf{z}}^{1}$. With similar notations, $I ! J ! \widetilde{G}_{I J}$ represents $\widetilde{K}_{\mathbf{z}}^{I J}$ with respect to the normalized frame $\widetilde{\gamma}(\mathbf{z})$ of $E(\widetilde{\mathbf{T}})$.

Now we are prepared to prove the theorem. For sufficiency, let $\Phi$ be a unitary operator from $H_{\mathbf{z}}^{1}$ to $\widetilde{H}_{\mathbf{z}}^{1}$ intertwining $K_{\mathbf{z}}^{I J}$ and $\widetilde{K}_{\mathbf{z}}^{I J}$ whose representing matrix with respect to $\gamma(\mathbf{z})$ and $\widetilde{\gamma}(\mathbf{z})$ is denoted by $U$. Then $U$ is a unitary matrix as both frames are normalized at $\mathbf{z}$. Moreover, the intertwining property gives

$$
I ! J ! G_{I J} U=U\left(I ! J ! \widetilde{G}_{I J}\right)
$$

that is

$$
G_{I J}=U \widetilde{G}_{I J} U^{*}
$$

for all $1 \leq|I|,|J| \leq k-1$ at $\mathbf{z}$. 
Observing that at $\mathbf{z}, G_{00}=\widetilde{G}_{00}=I_{n}$ and $G_{I 0}=\widetilde{G}_{I 0}=\mathbf{0}$ whenever $|I| \neq 0$, identity 3.4 holds for all $0 \leq|I|,|J| \leq k-1$, which gives

$$
\left[G_{I J}\right]_{0 \leq|I|,|J| \leq k-1}=\left(U \otimes I_{L}\right)\left[\widetilde{G}_{I J}\right]_{0 \leq|I|,|J| \leq k-1}\left(U^{*} \otimes I_{L}\right)
$$

where $U \otimes I_{L}$ denotes the diagonal block matrix with $U$ lying on all diagonal blocks. Taking inverse we get

$$
\left[H_{I J}\right]_{0 \leq|I|,|J| \leq k-1}=\left(U \otimes I_{L}\right)\left[\widetilde{H}_{I J}\right]_{0 \leq|I|,|J| \leq k-1}\left(U^{*} \otimes I_{L}\right)
$$

Specifying (3.6) block-wise we see that at $\mathbf{z}$,

$$
H_{I J}=U \widetilde{H}_{I J} U^{*}
$$

holds for all $0 \leq|I|,|J| \leq k-1$. Recall that $H_{I J}=\partial^{I} \overline{\partial^{J}} H_{00}$ and $\widetilde{H}_{I J}=\partial^{I} \overline{\partial^{J}} \widetilde{H}_{00}$, the sufficiency follows from combining (3.7) and Theorem 3.3.

Conversely, if $H_{\mathbf{z}}^{k}$ and $\widetilde{H}_{\mathbf{z}}^{k}$ are unitarily equivalent, then Theorem 3.3 implies the existence of a constant unitary matrix $U$ such that (3.7) holds, which in turn gives, by reversing the above arguments, the intertwining property (3.4). So the unitary operator represented by $U$ with respect to $\gamma(\mathbf{z})$ and $\widetilde{\gamma}(\mathbf{z})$ implements the unitary equivalence of $\left\{K_{\mathbf{z}}^{I J}, 1 \leq|I|,|J| \leq\right.$ $k-1\}$ and $\left\{\widetilde{K}_{\mathbf{z}}^{I J}, 1 \leq|I|,|J| \leq k-1\right\}$.

\section{References}

[1] L. Chen and R. Douglas, A local theory for operator tuples in the Cowen-Douglas class, Adv Math. 307 (2017), 754-779.

[2] M. Cowen and R. Douglas, Equivalence of connections, Adv. Math. 56(1985), 39-91.

[3] M. Cowen and R. Douglas, Complex geometry and operator theory, Acta. Math. 141(1978), 187-261.

[4] M. Cowen and R. Douglas, Operators possessing an open set of eigenvalues, Functions, series, operators, Vol. I, II (Budapest, 1980), Colloq. Math. Soc. Janos Bolyai, vol. 35, North-Holland, Amsterdam, 1983, 323-341.

[5] R. Curto and N. Salinas, Generalized Bergman kernels and the Cowen-Douglas theory, Amer J. Math. 106(1984), 447-488.

[6] P. Deb, On unitary invariants of quotient Hilbert modules along smooth complex analytic sets, arXiv:1708.06964 v2.

[7] R. Douglas and G. Misra, Equivalence of quotient Hilbert modules. II, Trans. Amer. Math. Soc. 360 (2008), 2229-2264.

[8] R. Douglas, G. Misra and C. Varughese, On quotient modules - the case of arbitrary multiplicity, J. Funct. Anal. 174 (2000), 364-398. 
[9] R. Douglas and V. Paulsen, Hilbert modules over function algebras, Pitman research notes in mathematics, Longman Scientific and Technical, 1989.

[10] D. Dokovic and C. Johnson, Unitarily achievable zero patterns and traces of words in $A$ and $A^{*}$, Linear Algebra and its Applications, 421(2007), 63-68.

[11] K. Ji, C. Jiang, D. Keshari and G. Misra, Rigidity of the flag structure for a class of Cowen-Douglas operators, J. Funct. Anal. 272(2017), 2899-2932.

[12] M. Martin, Hermitian geometry and involutive algebras, Math.Z, 188(1985), 359-382.

[13] M. Martin and N. Salinas, Flag manifolds and the Cowen-Douglas theory, J. Operator Theory, 38(1997), 329-365.

[14] G. Misra and A. Pal, Curvature inequalities for operators in the Cowen-Douglas class and localization of the Wallach set, J. Anal. Math. 136 (2018), 31-54.

[15] G. Misra and Md. R. Reza, Curvature inequalities and extremal operators, Illinois J. Math. 63 (2019), 193-217.

[16] B. Sz-Nagy and C. Foias, Harmonic analysis of operators on Hilbert space, North Holland, 1970.

[17] C. Pearcy, A complete set of unitary invariants for operators generating finite $W^{*}$ algebras of type I. Pacific J. Math. 12 (1962), 1405-1416.

[18] C. Pappacena, An upper bound for the length of a finite-dimensional algebra, Journal of Algebra, 197 (1997), 535-545.

[19] W. Specht, Zur Theorie der Matrizen II, Jahresbericht der Deutschen MathematikerVereinigung, 1940.

[20] K. Wang and G. Zhang, Curvature inequalities for operators of the cowen-douglas class, Israel Journal of Mathematics, 222 (2017), 279-296.

Li Chen

School of Mathematics

Shandong University

Jinan 250100, China

Email: lchencz@sdu.edu.cn 